\title{
Comer o no comer ¿es esa la cuestión?: una aproximación antropológica al estudio de los trastornos alimentarios
}

\author{
Mabel GRACIA-ARNAIZ \\ Medical Anthropology Research Center - Universitat Rovira i Virgili \\ mabel.gracia@urv.cat
}

Recibido: 08-06-2013

Aceptado: 26-02-2014

\begin{abstract}
Resumen
En este artículo se plantea la pertinencia de aplicar una mirada socioantropológica en el estudio de los denominados TCA (trastornos de la conducta alimentaria) con el objetivo de explorar vías para comprender la trama social que sustentan prácticas alimentarias en apariencia singulares, y a menudo calificadas desde la biomedicina, de extremas e ilógicas. La mayoría de los trabajos realizados sobre los determinantes sociales de la salud y, en particular, sobre la influencia de éstos en el estado nutricional y/o psicológico de las personas son de carácter epidemiológico y, aunque han dado luz sobre la prevalencia y evolución de ciertas enfermedades, explican de forma simple o insuficiente las dimensiones socioculturales de los trastornos alimentarios, tanto en términos de causalidad como de alcance. En este sentido, el texto propone incorporar el enfoque etnográfico como recurso metodológico que permite articular subjetividades y contextos y, en esa medida, dar luz sobre las estrechas relaciones entre comida, género y cuerpo. Se muestra que la principal aportación de la etnografía al estudio de la anorexia nerviosa proviene de abordar de forma contexualizada y relacional las concepciones y experiencias de los diversos actores que intervienen en el proceso de salud/atención/enfermedad, así como las interacciones que se producen, particularmente, entre pacientes y profesionales sanitarios..
\end{abstract}

Palabras clave etnografía, enfoque relacional, anorexia nerviosa, cultura, género

\section{To eat or not to eat, is that the question? An anthropological approach to the study of eating disorders}

\begin{abstract}
This article presents an argument for the relevance of the anthropological gaze in the study of eating disorders. Anthropology offers ways of understanding the social forces underlying food and eating practices that defy convention and are treated as irrational in biomedical discourse. Most studies of the social determinants of health, especially those dealing with their influence on nutritional and/or psychological status, are epidemiological, and although they shed light on the prevalence and course of eating disorders, they tend to offer overly simplified or insufficient explanations of the sociocultural dimensions of the problem, in terms of both its causes and its consequences. This article offers a holistic approach to the analysis of eating disorders that joins subjectivity to context. The sources of data used to develop answers to the question of how food, the body, and women are linked in eating disorders are life stories, group interviews, Internet blogs, and direct observation. Ethnography's main contribution lies in its contextualized and relational approach to the conceptions and experiences of the
\end{abstract}


various actors involved in the health/illness/treatment process, especially in interactions between patients and health professionals.

Key words: ethnography, relational analysis, eating disorders, culture, gender

\section{Referencia normalizada}

Gracia-Arnaiz, M. (2014). “Comer o no comer ¿es esa la cuestión?: una aproximación antropológica al estudio de los trastornos alimentarios”. Política y Sociedad, Vol.51 Núm. 1 73-94

Sumario: Introducción. 1.Una mirada distinta, pero no distante. 2.La cultura: una china en el zapato. 3.Conclusión. Bibliografía 


\section{Introducción}

En campos como los denominados trastornos de la conducta alimentaria (TCA) ${ }^{1}$, como sucediera hace un par de décadas en las drogodependencias (Romaní, 1995), se ha producido un fenómeno de medicalización, es decir, de inclusión en el modelo médico de determinados comportamientos, dándoles un estatus de enfermedad mental (Gracia y Comelles, 2007). Estas entidades clínicas son, junto a las adicciones a sustancias psicoactivas, unas de las que mayor alarma social producen en la sociedad española. Los trastornos alimentarios se conciben como psicopatologías caracterizadas por la alteración de la conducta en la ingesta y comportamientos de control de peso provocadas, principalmente, por el miedo a engordar. En la medida que afectan mayormente a mujeres jóvenes y que, en casos extremos, pueden ser graves e incluso causar la muerte, a lo largo de los últimos veinte años se han ido estableciendo criterios de asistibilidad en base a esta particularidad sintomática.

Sabemos, sin embargo, que las ideas respecto a lo que en un momento dado se concibe como enfermedad dependen de las experiencias e intereses de los distintos actores sociales y grupos dominantes, de tal forma que los significados dados y las interpretaciones hechas sobre las prácticas alimentarias y corporales son, en realidad, múltiples (Way, 1995). Conforman, ciertamente, una producción constante de categorías clasificatorias, de taxonomías y de descripciones de síndromes; también de procesos colectivos de toma de decisiones, de desarrollo de saberes y experiencias particulares que pueden ser transmitidos de una generación a otra y, en algunas sociedades como la nuestra, a profesionales especializados y dispositivos institucionales y modelos complejos de organización de la atención sanitaria (Comelles, 1988). En cierto modo los procesos de salud/enfermedad/atención son como los actores sociales los ven, escuchan y viven.

Este artículo tiene como objetivo principal mostrar la conveniencia de reconocer, precisamente, la diversidad interpretativa como medio para mejorar la comprensión y abordaje de los trastornos alimentarios. Para ello, en la primera parte del texto se propone aplicar una mirada distinta que no distante, planteando qué oportunidades de estudio proporcionan estas dolencias a la antropología y, al revés, qué puede ofrecer una aproximación holística, comparativa y diacrónica al conjunto de las disciplinas biomédicas que se han ido legitimando para atenderlas. La construcción de los TCA como enfermedades mentales ha implicado, a menudo, centrar la atención en la causalidad individual -factores biopsicológicos- en detrimento de las razones socioculturales, responsabilizando al sujeto de su aflicción y recuperación a menudo a través de actitudes monológicas (Martínez-Hernáez, 2008). Frente a un

\footnotetext{
${ }^{1}$ Aunque se ha criticado el uso antropológico de la etiqueta de TCA, en tanto que reproduce la clasificación psiquiátrica y la praxis médica, aquí se utiliza expresamente para destacar la presencia de unos diagnósticos que tienen efectos específicos en la problematización sanitaria y social de la alimentación.
} 
modelo centrado en establecer qué síntomas son característicos de cada trastorno y qué rehabilitación psicológica y nutricional es la más adecuada para normalizarlos, la etnografía viene proponiendo desde hace años estudiar las prácticas alimentarias y corporales en apariencia extremas - comer mucho, poco o nada- dentro de las situaciones en que toman sentido (Prince, 1985; Lester, 1995; Counihan, 1999). Para ello, incorpora una doble mirada desde dentro y fuera de la red asistencial, así como instrumentos de análisis que permiten observar los diversos espacios de interacción, escuchar a los distintos actores sociales y articular subjetividades y contextos.

En la segunda parte del artículo, se muestra que algunas de las dificultades para tratar estas aflicciones son consecuencia de una comprensión reduccionista de los conceptos de cultura, género y alimentación. Con frecuencia, para la biomedicina los factores socioculturales se entienden sólo como agentes específicos causantes de enfermedad/muerte que pueden ser abordados aisladamente (Lupton, 1994). En el caso de la anorexia nerviosa se concretan, sobre todo, en las dietas restrictivas y una actividad física desmesurada con el objetivo de adelgazar o no engordar. Las razones culturales quedan definidas exclusivamente en torno a los apremios que la sociedad lipófoba exige a las mujeres para mantenerse en la delgadez corporal, y todos los esfuerzos terapéuticos, cuando se dan, están orientados a conseguir el normopeso, la incorporación de la dieta óptima y la modificación de los modelos de "belleza saludable". Apenas se consideran las experiencias contadas por las pacientes que se quedan al margen de razones identificables para el diagnóstico y que, sin embargo, también se canalizan a través de prácticas alimentarias y/o corporales particulares (Gracia-Arnaiz, 2013). Se defiende, finalmente, que esta desconsideración podría explicar, en parte, el bajo éxito terapéutico.

\section{Una mirada distinta, pero no distante}

Por sus múltiples dimensiones, el fenómeno de los TCA se ha convertido en objeto de estudio para numerosas disciplinas. La literatura médica es la que se ha empleado más a fondo en la definición clínica de estos trastornos ya desde el siglo XVII, y si algo ha dejado claro dicha literatura es que estamos ante enfermedades que se caracterizan por su gran maleabilidad, en el sentido de que las manifestaciones asociadas varían bajo la influencia de factores históricos o de circunstancias sociales cambiantes (Rusell, 1985). Esta constatación ofrece oportunidades de estudio también para las ciencias sociales.

\subsection{De disciplinas y abordajes}

Los trastornos alimentarios, aún reconociéndose su carácter histórico (Brumberg, 1988; Bell, 1985), se plantean como patologías relativamente "nuevas" donde la cultura adquiere un papel explicativo relevante. Se considera que el origen de la 
anorexia o bulimia nerviosa, o mejor su incremento, está vinculado a determinados cambios sociales, económicos y políticos acaecidos en las sociedades occidentales contemporáneas. Dicha vinculación, lógicamente, ha favorecido en los últimos años nuevas oportunidades de estudio y un aumento de la literatura socioantropológica dando lugar a diversos abordajes analíticos. Aún tratándose de estudios con intereses dispares han servido, cuando menos, para relativizar el carácter anómalo de comer mucho/poco/nada, así como también para evidenciar que el cuerpo no es nunca simplemente biológico o social. Del mismo modo, ponen de manifiesto la necesidad de contextualizar la emergencia y la evolución de las enfermedades según las variables tiempo/espacio y, finalmente, son útiles para preguntarse por qué, en un momento dado, ciertos fenómenos se problematizan y otros no.

En base a esta literatura se pueden establecer cuatro tipo de aproximaciones dependiendo de los énfasis dados y los recursos teóricos y metodológicos empleados (Gracia-Arnaiz, 2012). En primer lugar, están aquellos trabajos que asumen los presupuestos epidemiológicos sobre el origen causal y la evolución de los TCA, aportando a la biomedicina técnicas de análisis utilizadas por las ciencias sociales para profundizar en lo que, desde este ámbito, se consideran factores sociales determinantes. Por otro lado, se encuentran las investigaciones que ponen énfasis en la comparación y el enfoque histórico, dedicándose principalmente a dar cuenta de la variabilidad de representaciones, significados y prácticas en torno al cuerpo y la comida en épocas y culturas distintas. En tercer lugar, están los trabajos que se han interesado en analizar, por una parte, la distribución desigual de la delgadez o de la gordura en función de variables como el género, la clase social o el origen étnico y en relacionarla, por otro, con los factores estructurales que afectan a los sistemas alimentarios, las relaciones de poder que determinan el acceso a la comida y las condiciones de vida de los grupos sociales. Y finalmente, tenemos aquellos estudios que abordan las concepciones biomédicas, las praxis clínicas y las políticas públicas sobre los trastornos alimentarios, analizando su relevancia sanitaria y social.

Estos últimos trabajos señalan que la investigación sobre TCA ha crecido sustancialmente de la mano de la medicina, en sus vertientes epidemiológica y clínica, y de la psicología. Del análisis de esta literatura, tan profusa como diversa, destacan varios aspectos. Por un lado, la virulencia del fenómeno. Habiendo ocupado más que un interés relativo en salud mental, los trastornos alimentarios se presentan desde los años ochenta como afliccciones que avanzan con rapidez: las tasas se duplican o triplican en numerosos países occidentales en apenas dos décadas (Gordon, 2000). Por otro, se destaca la casi total unanimidad mostrada por expertos y autoridades sanitarias a la hora de considerarlas enfermedades provocadas casi exclusivamente por el miedo a engordar. Sorprende, en tercer lugar, la incongruencia entre las principales causas apuntadas (cambios culturales) y las medidas adoptadas (modificación de conductas individuales). Y, finalmente, se constata una rápida medicalización: en los últimos treinta años se han multiplicado exponencialmente las actividades científicas (congresos, foros, revistas especializadas, organizaciones, investigaciones), los tratamientos clínicos y farmacológicos y los servicios sanitarios especializados. 
Si bien la literatura biomédica es extraordinaria no ha resuelto, sin embargo, el problema de la causalidad (Gracia y Comelles, 2007), tan amplia como incierta. En un intento por eludir los modelos reduccionistas, los trastornos alimentarios se presentan como enfermedades psico-bio-sociales -en este orden-, cuya etiología no es fácil de establecer ni abordar. En ocasiones se explican por predisposición genética; otras veces, por falta de autonomía personal; otras por conflictividad familiar y, con mayor frecuencia, por seguir regímenes de adelgazamiento de forma incontrolada o irracional. De toda esta multicausalidad, la última es la que predomina. Quizá una parte de las dificultades por aclarar cómo interactúan los diversos factores señalados provenga de la misma construcción del concepto de TCA, traducido del término en inglés eating disorders, los cuales reducen clínicamente lo social y psicológico a conductas que, por alejarse más o menos de los patrones alimentarios aceptables, se etiquetan de desordenadas, extremas o irrefrenables y, por ello, directamente de patológicas. Esta ideación no sólo deja al margen los motivos estructurales relativos al género y la cultura que están detrás de estas prácticas y que originan las incertidumbres subjetivas, sino que determina los modelos de abordaje en la red asistencial. Esta cuestión es relevante porque con frecuencia los tratamientos -centrados en la restauración del peso, la rehabilitación nutricional y la reestructuración cognitiva- muestran un éxito más que relativo. Se han señalado no pocas dificultades para la valoración de la efectividad de las intervenciones terapéuticas, empezando por las críticas hechas al uso de términos como "abandono", "adherencia" o "cumplimiento" en tanto que muestran connotaciones pasivizantes sobre el papel que juegan las pacientes durante la intervención (Sirvent, 2009). Así, muchas veces la eficacia a la que alude la psicología clínica de corte cognitivo-conductual se basa generalmente en los pacientes que terminan el tratamiento sabiendo que, sin embargo, entre el 65\% y el $80 \%$ de quienes lo inician no lo aceptan o lo abandonan (Bados López et al., 2002). En el caso de la anorexia nerviosa, el fracaso del tratamiento psicológico se ha cifrado entre el 25 y 40\% (Bulik, 2007), lo cual explicaría, en parte, por qué muchas personas diagnosticadas buscan soluciones fuera del itinerario asistencial.

Al margen de la clínica, los trastornos alimentarios ponen en evidencia las paradojas asociadas al comer mucho, poco o nada, y proyectan de modo más transparente valores sobre ciertas formas de comportarse con la comida y con el cuerpo: la vida como un menú, el ayuno como autocastigo, el cuerpo como prisión... Por eso, cobra interés aplicar una mirada que aborde los sentidos dados a ciertas prácticas y que vaya más allá de considerarlas síntomas de enfermedades multicausales que responden arbitrariamente a un sinfín de factores biopsicosociales, muchas veces fáciles de formular pero menos de demostrar.

Como se ha señalado desde la antropología médica (Menéndez, 1990), el modo más elemental de conocer y analizar el proceso salud/enfermedad/atención pasa por su reconstrucción mediante la observación, el relato o la documentación de los procesos asistenciales o itinerarios terapéuticos de casos e individuos concretos. Esta es una metodología que utiliza la medicina desde la Antigüedad clásica y no es muy distinta de la que hemos elaborado los científicos sociales más recientemente 
(Haro, 2000). Sin embargo, hay una diferencia sustancial entre la aproximación clínica y la de los antropólogos: a los clínicos actuales el contexto no parece decirles nada puesto que se rigen hoy por teorías de la causalidad individuales y biológicas, mientras que para los antropólogos el contexto es el que permite comprender, interpretar y aún explicar cuanto concurre en torno a una situación de enfermedad.

Es muy importante este punto, puesto que las herramientas puestas en juego para contestar a las preguntas que motivan los estudios socioantropológicos no se limitan a la curiosidad intelectual, sino a aplicar simultáneamente una doble mirada externa/interna mediante una aproximación metodológica específica. La distancia crítica es particularmente relevante cuando se manejan instrumentos cualitativos, y se refuerza, como es este el caso, mediante el empleo de metodologías comparativas que intervienen en distintos planos: la comparación entre afectados o afectadas de distintos síndromes dentro de la esfera de los TCA, entre instituciones de asistencia y profesionales del sector público y privado, entre las perspectivas de distintos profesionales: psiquiatras, psicólogos clínicos, trabajadores sociales, enfermeras y aun gestores y, finalmente, la comparación con los datos procedentes de la investigación clínica y socio-sanitaria internacional.

\subsection{Aportaciones etnográficas para el estudio de los TCA}

Como hemos apuntado con anterioridad en otros lugares (Gracia, 2002; Gracia y Comelles, 2007; Gracia, 2009), la antropología ha supuesto una aproximación diferente a un tema dominado en España por aportaciones principalmente procedentes de la psiquiatría, la psicología clínica y la nutrición, en general poco abiertas a las ciencias sociales. Decimos en España, puesto que en la literatura internacional las aportaciones interdisciplinares con participación de antropólogos o sociólogos son legión. Esto se refleja tanto en los diseños de investigación como en las metodologías empleadas. Por eso, el perfil de nuestros estudios no se aleja en exceso de las aportaciones anglosajonas a esta problemática (DiNicola, 1990; Katzman y Lee, 1997) pero difiere de los diseños clínicos o epidemiológicos publicados por investigadores españoles (Fernández y Turón, 1998; Chinchilla, 2003), más centrados en ofrecer información sobre detección y diagnóstico, instrumentos de cribado o tratamientos y menos afines al despliegue de análisis cualitativos.

La aproximación etnográfica trata de incorporar aquello que habitualmente se obvia en este tipo de estudios. La revisión bibliográfica se plantea como un punto de partida para conocer cómo las disciplinas construyen sus formas de saber y es imprescindible cuando se trata de entender qué se ha convenido sobre la emergencia de los TCA o su causalidad. Conviene, en este sentido, tener en cuenta no sólo lo que dice la literatura biomédica o psicológica acerca de la falta de apetito, el hartazgo, el vómito, la purga o la pérdida de peso como síntomas de enfermedad, sino también las aportaciones que las ciencias sociales han efectuado en los últimos veinte años acerca de los lenguajes del cuerpo y la comida y del embodiment de las experiencias culturales. Ello facilita una aproximación transhistórica que relativiza 
el carácter enfermizo de ciertas prácticas, vislumbra el reduccionismo del discurso biomédico y descubre el papel de las lógicas culturales. Dentro de las técnicas de análisis cualitativas que permiten al etnógrafo aplicar una mirada en profundidad están las historias de vida (Giordiani, 2009; Moreno, 2010). Los relatos biográficos obtenidos en base a diversas entrevistas son herramientas muy útiles cuando lo que se persigue es conocer las experiencias y representaciones de personas diagnosticadas de una enfermedad que con frecuencia se cronifica y averiguar cómo éstas incorporan e interpretan sus padecimientos junto otros aspectos de orden contextual.

Considerar las experiencias de aquellas personas que han sido y son consideradas como anoréxicas o bulímicas es una vía privilegiada para tratar de comprender el porqué del autoayuno o el hartazgo y los límites del "retrato robot" con que la biomedicina homogeniza a menudo a sus pacientes. En el caso de los TCA, esta aproximación singulariza a las afectadas como mujeres adolescentes, de clase media, blancas, inteligentes, inmersas en ciertos tipos de familias (hiperprotectoras o conflictivas) y con perfiles psicológicos singulares (perfeccionismo, obsesión, autocontrol...). Sin embargo, aunque este retrato sirve para delimitar qué mujeres, de las que ayunan, están enfermas no deja nada claro por qué otras, que también apenas comen, no lo están. El modelo, arropado con todos sus instrumentos de detección, sirve para establecer quienes son pre-anoréxicas y decidir cuáles son exanoréxicas - una etiqueta que las perseguirá toda su vida- en base a la presencia o ausencia de síntomas concretos. Incluso, aunque con bastantes más dificultades clasificatorias, qué hombres sufren estos trastornos y cuáles no.

Todas estas consideraciones se manifiestan en las historias de vida. Los relatos bibliográficos muestran que algunas de pacientes se niegan a salir de su condición de anoréxica o bulímica, en tanto que interiorizan hasta tal punto su "trastorno" que evitan cualquier mecanismo de recuperación que pudiera extraerlas de su estatus de enferma: "La anorexia es que dejas de comer cada vez más alimentos... Cuando se es anoréxica se te va la regla, te miras a un espejo y te ves gorda, te aterroriza saber lo que pesas... Soy anoréxica restrictiva, es una enfermedad muy grave y me puedo morir. Por eso aquí nos vigilan todo el día. Aunque no nos dejan que nos veamos fuera del hospital, yo me he hecho varias amigas de aquí. Tengo una amiga con bulímia y otra comedora compulsiva, yo ahora soy anoréxica purgativa..." (MP, 17 Años). En el otro extremo, otras mujeres, una vez comprendida y reconocida la situación límite en la que se encuentran, buscan salida o se "dejan ayudar" no tanto a través de las instituciones de atención médica, en donde se resuelven los aspectos más formales del trastorno (ganancia de peso), como a través de sus propias redes sociales, las cuáles incluyen tanto a familiares, amigos y compañeros de estudio y trabajo, grupos de ayuda mutua, como a las propias amigas anoréxicas o bulímicas que, en mayor o menor medida, han superado las fases más críticas de la aflicción y han salido de la espiral en la que estaban: “"Había una chica de 12 años que ya había pasado por el hospital 5 veces. Siempre le habían hecho el mismo tratamiento. Había estado tres meses, dos meses... Todo era salir de allá y volver... Yo tampoco tengo la solución de lo que hay que hacer, pero lo que sí que puedo decir es que ésta no es la solución. A mi tampoco me funcionó 
especialmente bien, porque tuve una recaída y lo volví a pasar muy mal. Y mi miedo era que no me volviesen a encerrar allí... Yo creo que en estos casos lo más importante es hablar... que alguien te escuche, por eso el grupo de ayuda mutua fue mucho más útil” (PCA, 43 Años). Ciertamente, las historias de vida muestran que una parte significativa de los mecanismos utilizados por las mujeres para superar estos malestares se generan al margen de las unidades de atención y en las redes sociales informales o se producen de forma espontánea. De ahí, que sea oportuno conocer cómo funcionan estas vías de apoyo y autoayuda.

El objetivo de una antropología relacional es conocer también cuáles son las opiniones de los equipos de expertos que tratan con las pacientes, cómo definen estas enfermedades y a sus enfermas, cómo valoran sus métodos de diagnóstico y sus tratamientos, sus éxitos o fracasos. De ahí que sea necesario indagar en la construcción de las propias categorías nosológicas y de los procesos asistenciales, y, como no, en las relaciones entre pacientes y terapeutas. Esto puede hacerse incorporando, a pesar de las conocidas dificultades para aplicarla en el ámbito asistencial, la observación directa o participante. Junto a los relatos biográficos, las entrevistas en profundidad, los grupos focales o el diario de campo, y complementándolos, la herramienta observacional se hace imprescindible para contrastar el contenido de las interacciones allí donde se dan, ya que permite contemplar las relaciones entre personas diagnosticadas, profesionales sanitarios o familiares en los espacios en los que se producen y toman sentido (la consulta, los comedores, los talleres, las asociaciones, etc.) y escuchar, en los distintos contextos, todas las voces: "Observo que Judith no habla apenas y le cuesta separar la vista del plato. Tiene un trozo de calabacín y pescado, y se entretiene en quitarle el rebozado de harina y partirlo en trozos muy pequeños. Apenas se lleva el tenedor a la boca, y cuando lo hace tarda mucho en tragar. Al final se come el trozo de calabacín pero esconde parte del pescado debajo de la piel de la manzana que toma de postre. La otra enfermera está sentada a su lado. Se pone muy seria y se queda mirando fijamente el contenido de su plato cuando comienza a comerse la manzana, pero no le dice nada. Aparenta no darse cuenta. Cuando termina con la manzana, la enfermera retira la bandeja... Luego, en privado, habla con ella y me cuenta que la paciente tenía razón, que ha sido un fallo de la dietista o de cocina. No se han acordado del pacto que habían hecho con Judith. No le gusta nada, para nada la merluza" (Diario de campo MB, comedor de un centro de día).

Para contextualizar la diversidad de discursos y experiencias hay que averiguar qué se está haciendo en las redes de atención sanitaria, tanto a nivel público como privado: si los criterios de diagnóstico se están o no diversificando, si se incluyen otras técnicas de tratamiento que vayan más allá de la farmacología o la terapia individual o grupal, si se están creando unidades de tratamiento diferenciadas de otros trastornos mentales y por qué. Sabemos, por ejemplo, que la terapia narrativa que se está aplicando en instituciones asistenciales de otros lugares del mundo apenas tienen presencia en la sanidad pública española. Solo algunos centros, la mayoría de orientación sistémica, han incorporado tratamientos que van más allá de hacer entrevistas terapéuticas individuales o familiares o prescribir fármacos. Nos 
preguntamos si la razón de esta ausencia se debe, precisamente, a que es una herramienta que busca el acercamiento dialógico e interactivo de la terapia, y reconoce a los pacientes como personas expertas de su propia vida.

Para la etnografía, el análisis de los marcos interpretativos de los TCA, de sus síntomas y de su abordaje, se constituyen en un objeto de estudio relevante. Es muy interesante contrastar las ideas que unas y otros tienen del mismo problema. Para muchos clínicos "una muchacha anoréxica es siempre una persona con caracteres anómalos, excesivamente perfeccionista, con una autoestima muy baja y con dificultades para las relaciones sociales. Sufre, es compulsiva. Son extraordinariamente mentirosas. Niegan la evidencia, que están obsesionadas por el peso" (psicoterapeuta). La biomedicina es muy contundente a la hora de idear los "verdaderos" marcos interpretativos de estas aflicciones, pero dicha ideación se hace al margen de escuchar muchas voces disonantes: "Son de los cuadros psiquiátricos más complejos que existen. Hay más de 40 factores implicados que predisponen, agravan o disparan la enfermedad. Todo empieza por un régimen... Cuando la posible víctima entra en una espiral de pérdida de peso; cuando es sorda a todo tipo de consejo familiar; cuando le desaparece la regla y cuando niega todo problema achacándolo a la percepción de los demás...” (psiquiatra).

En relación con las mujeres diagnosticadas hay que saber si ellas perciben que están enfermas o no, cómo definen e interpretan su enfermedad, de qué modo les afectan los tratamientos administrados o si les alivian o agravan sus malestares: "no había forma de que te visitara más de dos veces el mismo psicólogo. Cada dos por tres tenías que explicarte tu vida a un tío diferente. Te encuentras que si uno hace prácticas, que si otro rota... No siempre te apetece estar contando tus historias a un tipo o tipa que sabes que de aquí dos días si te he visto no me acuerdo. Esto no ayuda, al contrario". Es relevante saber que, a pesar de los acuerdos con los profesionales sanitarios, las pacientes se saltan las normas e engañan a sus terapeutas: "Hablé con Beatriz [psicoterapeuta] y me dijo que si quería vivir o morir y que si seguía perdiendo peso me iba a hacer la gráfica.. Me han hecho la gráfica y si bajo más peso me quedaré sin visitas el fin de semana y me lo tendré que comer todo a la fuerza...No he visto a mis padres porque estoy condicionada...He estado hablando con Beatriz sobre mi enfermedad y me ha dado unos tests para hacer, los de siempre, para ver si distorsiono la imagen... Me ha dicho que no le mienta" (PM, 28 A, diario). En este sentido, hay que conocer cómo van posicionándose las mujeres y los expertos en toda esta caracterización, porque en numerosas ocasiones las visiones no siempre coinciden: "nunca me he sentido como el común de las mujeres. Jamás en mi vida he ido a un gimnasio. No me depilo, no me maquillo, no uso tacones, no sé lo que es "arreglarse" si no es ponerse un par de pinches y un moño. No práctico ningún rito absurdo supuestamente necesario para vernos más bellas. Tampoco disfruto las reuniones femeninas en las que se termina hablando de hombres. Tampoco me considero una señorita. A ver si me lo aclaran. ¿Qué es ser femenina hoy en día?” (M, bloguera).

El contraste entre las visiones de los profesionales de la salud y las de las pacientes evidencia las dificultades y las limitaciones que el modelo biomédico tiene para 
interpretar la lógica que rodea a ciertas prácticas sociales. Esto da lugar a dificultades reconocidas por los clínicos para tratar a los pacientes y, lo que es más importante, para prevenir estas enfermedades. Por eso, una tarea por hacer es valorar las consecuencias de la falta de diálogo entre esas dos lógicas narrativas. A pesar de las limitaciones, hay que tratar de soslayar las visiones más estereotipadas y recurrentes que se ofrecen desde la literatura médico-psiquiátrica o psicológica, y buscar alternativas que den respuesta a los diversos porqués que rodean estas aflicciones. $\mathrm{Y}$ eso solo puede hacerse discutiendo acerca de los límites y ventajas que proporcionan ciertas herramientas como las entrevistas o la observación que todos los estudiosos usamos, aunque sea de muy distinta manera.

Si el trabajo etnográfico en instituciones especializadas, en asociaciones o grupos de ayuda o en pisos tutelados permite profundizar en la relación entre la contextualidad y la subjetividad, entre la realidad objetiva y su interpretación individual, otro espacio que para la antropología se presenta como una oportunidad irrenunciable es internet. Los foros y blogs dispuestos en la red recogen narrativas elaboradas por personas que se autodefinen como anoréxicas o bulímicas con reflexiones de extraordinario interés. Internet ha planetarizado la experiencia de la enfermedad en la medida que son infinitos los foros de discusión y la aportación de información sobre prácticamente todas las patologías y en todas las lenguas. Esto significa que el llamado proceso de globalización conduce a la posibilidad de compartir experiencias muy alejadas y de estructurar espacios micro-sociales virtuales. Al mismo tiempo ofrece un abanico relevante de recursos en los procesos de construcción y de deconstrucción de categorías y taxonomías nosológicas.

Por ello parece oportuno no sólo interpretar qué sucede con las nuevas formas de asociarse y participar que tienen las personas diagnosticadas de una enfermedad, sino conocer cuáles son los sentidos que estas relaciones generan, que características tienen los nuevos puntos de encuentro y cómo es el proceso de identidad que se forma entre los miembros. Las comunidades virtuales resultan un campo valioso para estudiar cómo se agrupan las personas en torno a las emociones, cómo desarrollan lenguajes y comunicaciones y cómo navegan sus relaciones entre la virtualidad y la realidad sin complicaciones: "A mí, me gusta escribir para luego releerme. Veo más en frío las cosas. No me vale con pensarlas, es...distinto. El hacer público ese escrito es una mezcla de no querer seguirse ocultando, con buscar comprensión con tendencias de gremio, digo yo. Y con darle un rollo más "real", creo. Me gusta saber quiénes me leen (aunque no comenten siempre, claro). $Y$ me gusta ver otras opiniones sobre los temas que yo hablo. Refunfuño ante los lectores anónimos. Pero no me quejo de que me lean. Me quejo de que me lean en silencio y no píen" (A, bloguera).

Por otro lado, hay que considerar la comunidad virtual como un grupo de personas que no sólo se identifican por aquello que les une, en este caso los trastornos alimentarios, sino porque muestran formas de resistencia simbólicas, luchas contrahegemónicas y defensa de espacios culturales con autonomía relativa capaces de reivindicar la diferencia y de vivir como transgresores en las fronteras mismas de la marginalidad médica, negando la norma social en coherencia con las opciones 
identitarias de sus vidas: "Me pregunto porque mierda la gente piensa que uno hace esto enteramente por vanidad, si supieran la esencia de todo esto, esto es algo mío, tan mío, que voy a defenderlo con uñas y dientes, y si me cuesta la vida, me sentiré mas que recompensada (...) porque para mi la anorexia no es sinónimo de enfermedad, simplemente es una manera de vivir mi vida (...) jamás permitiré que se metan con algo enteramente mío como son mis ayunos, vómitos y dietas" (I, bloguera.). Según Ledo (2013), el hecho de ser diagnosticada de anorexia o bulimia conlleva una condición en sí misma que -para las personas afectadas- tiende a tomar relevancia e imponerse como escenario de lo actual ocultando con frecuencia el problema raíz, que en muchos casos está lejos del eje central, desvirtuándose, e incluso permaneciendo oculto. Esta es la razón que hace que, en muchos casos, una persona escriba su diario y lo haga público como sucede en la blogosfera.

$\mathrm{El}$ auge de testimonios en la red coincide con el hecho que para el modelo hegemónico, y a diferencia de otros enfoques como el sistémico o el psicoanálisis, las pacientes apenas si tienen voz y sus narrativas orales y/o escritas no son para estos terapeutas primordiales aunque, en ocasiones, las inciten a escribir. Sus diarios son escritos para ser leídos por ellas mismas y para que eso les sirva de ejercicio autoreflexivo y menos para ser tomados como una herramienta interactiva en la terapia. Sin embargo, los relatos biográficos dispuestos en los blogs de Internet muestran claramente que estas mujeres tienen una necesidad de "hablar" con los demás, incluso con sus propios terapeutas a los que refieren a menudo: "Extraño a mi primera psicóloga. No quiero volver a ninguna a excepción de ella (...) estuvo en el momento más importante de mi enfermedad: el principio...cuando yo NO era muy consciente y no sabía nada, en comparación a ahora. Momento clave, por cierto...si las 2 hubiésemos sabido lo que se venía se habría podido hacer mucho, mucho más! Era preciosa, siempre olía bien, su pelo era brillante y perfecto...su ropa tenía onda, siempre flaca, pero muy vital...Me dijo cosas que nunca he olvidado (a diferencia de la última que tuve y de mi psiquiatra que es una verdadera $M-I-E-R-D-A \ldots$ (A, bloguera). Necesitan hablar fuera del espacio institucional, comunicar experiencias y emociones que raramente son explicadas en las sesiones terapéuticas. Las mujeres dicen que la red les otorga la libertad de expresión que no tienen o sienten en las consultas. Tal como plantea Ledo (2013), el análisis del mundo virtual permite descubrir aquello que es común, lo que resulta idéntico en todos los casos, no importa si es la auténtica realidad, si somos producto de una realidad virtual, o si es el producto de una computadora. En cualquier circunstancia, lo que es importante para el sujeto es el acto de la experiencia en sí misma. No podemos demostrar con toda seguridad "qué es real”, pero si podemos descubrir cómo algo "llega a ser real" para nosotros. La etnografía virtual se fortalece precisamente por su falta de recetas (Hine, 2004:23).

\section{La cultura: una china en el zapato}


Si la aproximación etnográfica muestra la diversidad de las experiencias y significados construidos por los actores sociales, también permite reflexionar sobre el papel de la cultura en tanto que factor causal de los trastornos alimentarios. En la medida que la etnografía se define por el esfuerzo de desgranar las tramas de significación construidas entre sujetos y contextos a través del uso de herramientas cualitativas, podría convertirse en un recurso compartido entre quienes se dedican al estudio integral de los trastornos alimentarios. La minimización de lo cultural en ciertos modelos explicativos se debe a la desconexión entre las diferentes aproximaciones científicas, principalmente entre ciencias biomédicas y socio-sanitarias y, porque sólo las primeras pretenden estar legitimadas científicamente para definir y abordar las enfermedades y a sus pacientes. Nos preguntamos hasta qué punto los profesionales sanitarios pueden, deben o saben hacer diagnósticos sobre la realidad social y sobre su influencia en la emergencia de estas aflicciones, hasta qué punto los diagnósticos realizados sobre las causas culturales son acertados y hasta qué punto se ofrecen soluciones plausibles para su modificación.

\subsection{De sujetos y contextos}

Como señalábamos al principio, buena parte de los profesionales sanitarios reconocen unánimemente el papel de la cultura en la causalidad de los TCA (Garner y Garfinkel, 1980; Toro, 1996), pero de un modo que no puede emplearse como concepto analítico ni recoge su complejidad. Esto es así porque la concepción de cultura que maneja la biomedicina oscila entre un sinónimo de “educación” -de aprendizaje de competencias académicas o cívicas-, y una ingenua y anacrónica definición como conjunto de rasgos que, articulados unos a otros, etiquetan una determinada identidad "étnica” (afro-americano), o "cultural” (francés). Con dificultades para ir más allá, la retórica biomédica se fija en lo que conoce, las trayectorias clínicas, y desde la clínica valora la responsabilidad del paciente en su enfermedad.

En la actualidad, la tendencia a convertir en patología cualquier malestar, incluso banal, se explica en parte por el desmantelamiento de los sistemas de soporte social incompatibles con el individualismo del modelo societal en el que vivimos, y por la delegación sobre los profesionales (o expertos) de las responsabilidades de su gestión. Considerar patología cualquier forma de desviación o transgresión social exculpa de su causalidad a los agentes socioeconómicos o políticos, permite abordarla individualmente y culpabilizar a la víctima de su propio mal, unas veces por su constitucional vulnerabilidad, otras porque "se lo ha buscado". En las narrativas de los clínicos es muy común que se responsabilice al paciente o a su entorno más inmediato de sus males (Gracia-Arnaiz, 2013). Comer mucho o poco es una elección personal, hacerlo ordenada o desordenadamente también. Si enferman, es su responsabilidad: "Se lo buscan ellas solitas. Todas empiezan igual. Se ponen a dieta, empiezan a pesarse cada día... hasta que pierden el control" (psicoterapeuta). 
Esta idea también es recurrente en los relatos de las mujeres que reciben tratamiento: "No sé cómo he llegado hasta aquí. Todo parecía un juego. Parecía tener el control, parecía que cuando yo deseara diría un "hasta luego" para aparcar mi lucha con la comida (...) Pero no es así.” Las personas diagnosticadas se describen como sujetos que no saben que están enfermos hasta que el médico las diagnostica: "Aprendes a ser anoréxica. Los psicoterapeutas te dicen que estás enferma y que estás en un psiquiátrico y que vas a aprender a estar en él... Aprendí a desconectar. Me comportaba como se suponía que ellos querían que me comportase... Me pasaba el día en la cama, en cuclillas... llegué a creerme que era pequeña, una niña. También aprendes de tus compañeras, unas de las otras. Las que llevan más tiempo dentro te enseñan a esconder la comida, te dicen que has de hacer para que cuando te peses, peses más... Yo veía que sí, que hacía lo mismo que mi compañera que era anoréxica, vomitaba, la miraba a ella y yo hacía lo mismo...” (MA, 35A). A partir de aquí, una conciencia de anormalidad muy bien delimitada se instaura en sus vidas y el discurso de la enfermedad empieza a adquirir forma en su mente y en su cuerpo, hasta tal extremo que se buscan y se recrean en ella: sus dolencias están más o menos definidas clínicamente, y las consecuencias de la malnutrición aun mejor. También las vías para solventar sus problemas. La mayoría de sus agobios y manías se explican como consecuencia del no comer o del comer compulsivamente porque, efectivamente, tiene consecuencias devastadoras psíquicas y físicas. Y sobre estas consecuencias se actúa en la mayoría de consultas: "Piensa -le insistió su psicóloga- que te has de meter en la cabeza este régimen, con independencia de que tengas más o menos hambre, es muy importante no saltarte comidas. Para que te cures, la comida ha de ser tu prioridad, tan importante o más que las medicinas que te tengas que tomar" (piscoterapeuta).

El enfoque hegemónico se centra en el sujeto y en los aspectos físicos y psicológicos que deben ser tratados. Entonces establece los recursos terapéuticos mediante una combinación de rehabilitación nutricional, recuperación de peso y psicoterapia a largo plazo (Turón, 1996). Algunos centros se consideran ya efectivos si logran hacer que sus pacientes ganen peso y les dan alta cuando todavía siguen teniendo las mismas ideas sobre la comida o el cuerpo que antes de ingresar o iniciar la terapia. Lo único que las "saca" de su enfermedad y de su ingreso es el haber aumentado el peso hasta los límites de normalidad y demostrar que la distorsión de la imagen es menor que cuando empezaron el tratamiento: "sólo querían que llegásemos al peso y ya está. Pura y simplemente eso. Cuando consideran que esa persona está en el peso, te daban el alta. Sin ninguna explicación de qué peso tienes, de qué peso has de tener, sin ninguna explicación. Simplemente, de tanto en tanto pasaba el médico y montaba el cirio. ¿Qué has hecho? ¿Por qué has bajado de peso? ¿Cuándo vas a aprender a comer y a hacernos caso? Y te castigaban quitándote las visitas del fin de semana" (BA, 27 años). No se consideran las relaciones entre los padecimientos subjetivos y el entorno más o menos inmediato. Si para un científico social ir del contexto al caso es relativamente fácil, al clínico le es muy difícil pasar del caso al contexto, puesto que los más carecen de los instrumentos metodológicos indispensables para comprenderlo. Por eso la biomedicina acepta esa con- 
cepción difusa de "la sociedad" o "la cultura" como algo lejano, inconcreto, el argumento final cuando fallan las interpretaciones biológicas. Esa actitud impide un análisis profundo de los efectos del sistema sobre los individuos.

Cuando en las primeras décadas del siglo XIX los efectos colaterales del capitalismo eran la insalubridad, las enfermedades infecto-contagiosas, las enfermedades laborales o los accidentes de trabajo, la respuesta médico social fue asumir su responsabilidad para cambiar las condiciones de trabajo o de vivienda. Lo hizo meritoriamente (Comelles, 1988). En los siglos XX y XXI, esos profesionales tienen dificultades para reubicarse ante una demanda de enfermedades crónicodegenerativas o de malestares que responden más a una causalidad basada en la interacción entre los seres humanos y el entorno que a la biología. Articular la experiencia clínica con procesos estructurales y dinámicos vinculados a las desigualdades sociales y de géneros, la medicalización o la mercantilización del cuerpo y salud en tanto que estrategias a largo plazo del capitalismo es demasiado abstracto para un diagnóstico clínico y una orientación terapéutica sobre un paciente singular. No se comprende, por ejemplo, que tanto el comer hasta hartarse como el comer poco o nada revisten significados que van más allá de nutrirse. Para hacerlo, deberían tener en cuenta la polifuncionalidad de las prácticas alimentarias y ubicarlas en los contextos experienciales y emocionales en los que adquieren sentido, aquellos que Appadurai (2001) llama etnoscapes y que, lejos de ser rígidos y estables, son el producto de las permanentes hibridaciones culturales. Por eso asombra una sociedad opulenta en la que algunas personas deciden ayunar para tener una apariencia aceptable, o hartarse y luego vomitar o purgarse para lo mismo. Durante la actual fase de capitalismo post-fordista la biomedicina - y la psiquiatría-, se ven incapaces de analizar sus efectos colaterales puesto que en él son fundamentales la producción de bienes y servicios simbólicos, y no materiales como antes. Por eso hay una discrepancia profunda entre la actitud de la biomedicina ante las enfermedades de raigambre biológica, entre las que se siente segura, y su desconcierto ante las que solo parcialmente puede reducirse a ello.

Carecer de una mirada que permita una lectura global de los procesos implicados, y rechazar las teorías que lo hacen -como por ejemplo la psicoanalítica- por su carácter puramente especulativo, impide a la biomedicina dar cuenta de esta interacción. Atrapados por la lógica utilitarista del modelo médico y por una práctica, cada vez más basada en protocolos diagnósticos y terapéuticos rígidos, los discursos de psiquiatras, psicólogos y en general de los profesionales del " $p s y$ " deben formularse de modo aparentemente neutral y ser impermeables a los juicios morales y a los valores culturales y lo más próximos posibles a la lógica discursiva de la biomedicina (Martínez-Hernáez, 2008). Así pueden diseñarse protocolos afines, nutridos por un imponente dispositivo diagnóstico y terapéutico. Su inconveniente es que no tratan de comprender, sino solo de identificar para "tratar". Esto les exime de involucrarse en la subjetividad del paciente, de controlar las relaciones de intersubjetividad entre ambos y de cualquier forma de compromiso social o político con las condiciones o factores que pudieran producir esos malestares. No es necesario identificar las "circunstancias" del paciente en su vida cotidiana, solo etiquetarlas 
desde fuera mediante proyecciones y valores comunes que los profesionales comparten, en buena medida, con el conjunto de la ciudadanía.

\subsection{Una concepción limitada de la alimentación, el cuerpo y el género}

El tipo de causalidad cultural que la biomedicina reconoce en los TCA se limita a reconocer factores que, como la lipofobia o temor a ganar peso, y la distorsión de la imagen corporal sostienen el edificio de la nosografía, ignorando los sesgos de género que han influido en ella. Se establece así un feed-back entre las categorías nosológicas construidas (los trastornos mentales) y la aceptación social de las enfermedades, reforzándose mutuamente. Si, como hicieron Bordo (1993) o Hepworth (1999), se analizaran los discursos sobre el género y las experiencias con la comida en las entrevistas clínicas, se podría observar cómo los hombres son candidatos menos probables a los TCA. De hecho, la literatura biomédica continua considerando los trastornos alimentarios como enfermedades de condición femenina. A menudo, los clínicos hablan de la anorexia masculina como mucho más severa, diferente a la femenina, a pesar de la similitud en los síntomas. Para ellos, la anorexia se explica como resultado de una malnutrición severa asociada a menudo a depresiones endógenas: "Normalmente se trata de situaciones diferentes. Las chicas que nos llegan suelen ponerse a dieta porque quieren adelgazar y parecerse más a las modelos, el problema es que se pasan de rosca... Los chicos, sin embargo, suelen preceder la fase anoréxica o combinarla con algún otro trastorno psiquiátrico, como una depresión... Sí que pueden hacer referencia al deseo de querer pesar menos, pero no es tan obvio como en el caso de las chicas” (psiquiatra). En cambio, la identidad de la mujer anoréxica o bulímica sería producto de una crisis perpetua, centrando el origen del conflicto en su inseguridad personal y su insatisfacción corporal. Aún así, estudios recientes señalan cada vez más causas "socioculturales” parecidas para ambos géneros, estableciendo como la más relevante la influencia mediática en la promoción de la delgadez (Toro et al., 2005).

Estas actitudes traducen, así, una concepción de cultura taxonomista que no tiene en cuenta los componentes estructurales y dinámicos de las prácticas alimentarias ni del sistema de género. Incluso las tesis culturalistas recogidas por la psiquiatría considerando que la cultura lipófoba tiene un papel precipitante o mantenedor de los TCA (Toro, 1996) son utilizadas, sobre todo, para "cerrar" las fronteras o los límites de los trastornos alimentarios, no sólo para ajustar unas taxonomías aparentemente neutrales, sino sobre todo, porque delimitando los síntomas únicamente a las sociedades "modernas" y "occidentales" y todos los “males" sociales en torno al desmesurado culto al cuerpo delgado se han podido perfilar mejor los factores y grupos de riesgo: "muchas chicas hacen dieta porque en su familia hay conductas alimentarias atípicas y por las presiones recibidas durante la infancia-adolescencia que valoran excesivamente la delgadez" (psicoterapeuta).

Estas tesis no han buscado, sin embargo, transformar las relaciones de poder y desigualdad que en estas sociedades se producen y que, en buena parte, podrían 
estar explicando la incorporación (embodiment) de ciertas prácticas corporales (dieting) y ciertos consumos alimentarios (restrictivos), por otro lado muy generalizados entre las mujeres (Zafra, 2007; Bernal, 2011). Esta es una tarea difícil para la que el experto (psiquiatra, psicólogo, nutricionista) no está preparado, ni en términos de competencia profesional ni posiblemente social. Referirse al "entorno" significa, nada más y nada menos, que a la organización misma de una sociedad, es decir, a los condicionantes económicos, culturales y políticos, en este caso, de un capitalismo de consumo que afecta a todo y a todos: a las relaciones de género, a los valores que priman el individualismo y el consumo, a las estructuras familiares a las formas de entender la salud y la enfermedad, a la cosificación del cuerpo (Gracia-Arnaiz, 2010). Estas características son, en su totalidad, de un orden cultural muy específico: el que se va desarrollando en los países industrializados desde principios del siglo pasado y cuyo modelo, por otro lado, se ha ido extendiendo por todo el mundo acompañando el fenómeno de la globalización económica y cultural. El contexto, en consecuencia, puede explicar, al menos en parte, por qué las mujeres, por qué la comida, por qué determinadas profesiones, por qué a determinadas edades. Una visión más amplia del "entorno" (de la cultura) y un enfoque holista y comparativo (de las prácticas alimentarias y corporales) permiten constatar que ciertos síntomas, como el dejar de comer, restringir la comida o hacerlo hasta hartarse, no son sólo propios de las sociedades industrializadas, ni particularmente de las mujeres, sino adaptativos o simbólicos, según las circunstancias.

Señalar los límites interpretativos del modelo biomédico es relevante en cuanto que tras las prácticas de ayuno, atracón, vómito o purga no siempre hay un deseo de ser o permanecer delgada. Asociados principalmente a mujeres jóvenes en las sociedades modernas, los TCA unas veces se han explicado por su mayor dependencia personal y emocional, por su falta de autoestima, por su incapacidad de comunicarse, por su inseguridad en sí mismas y, otras, por su inclinación a acatar más estrictamente los dictámenes de marketing y la moda. En ningún caso, se definen esas pacientes por sus maneras de ser y entender la vida o por su capacidad de "usar" algo tan significativo, y con tanto valor económico y simbólico en nuestra sociedad, como el cuerpo y la comida en tanto que formas de resistencia, de contestación o de desacato ante la profusión de normas familiares, culturales y/o sanitarias. Hemos visto que las experiencias de las pacientes, sus narrativas emic de síntomas, tampoco caben en este modelo. Tanto se han regulado y minimizado las causas contextuales, que se evitan investigar, por ejemplo, los significados personales/sociales del "dejar de comer" o "del hacerlo en exceso.

Quienes padecen trastornos alimentarios acostumbran a tomar el cuerpo como expresión de aflicción y como vehículo para manifestar malestares de diferente índole: De pequeña ya tengo un trauma con la comida porque a mí nunca me ha gustado nada y no tenía nunca hambre para comer, y la hora de la comida era para mí un infierno, ¡no quería! Ni sentarme a la mesa ni comer ni nada. Y todo obligadamente, todo muy mal, lo que eran las legumbres y muchas veces era comerlo y echarlo. No comía porque no me apetecía, no porque quisiera estar delgada ni nada. Siempre he sido muy rebelde y como mis padres son muy autoritarios, mi 
padre es muy autoritario, una manera de rebelarme era no comer. Cuando piensas cómo empecé con el trastorno alimentario, piensas: ipero si yo no he comido nunca! (BA, 27Años). Hay quienes dejando de comer pretenden borrar cualquier signo corporal de feminidad: “...que horror, ya me están saliendo tetas de nuevo no puede ser, no quiero, quiero ser niña siempre, quiero mi cuerpo de niña siempre, no quiero crecer nunca, no quiero engordar, no quiero tener esas curvas de mujer, quiero ser como esas chiquitas de 8 años todas flaquitas y enclenques, con mis muslos tan delgados que parezcan que se van a romper...” (internatua I). Pero también, de forma contraria, hacer dieta constituye un medio para ejercer una disciplina férrea sobre el cuerpo y la mente que produce enorme satisfacción (Van Dongen, 2000): "Si soy capaz de dejar de comer, con lo que me gusta, puedo demostrarme a mí misma de lo que soy capaz. Sólo hago dieta unos días. Pierdo los kilos que quiero y vuelvo a comer. Para mi es un sacrificio pasajero. Cuando el pantalón se abrocha sin problema siento tanto placer como cuando me como un bocadillo de jamón de jabugo" (CB, 30 años).

La extensión de estas aflicciones a otros tramos de edad no circunscritos a la adolescencia, a todas las clases sociales e incluso a sociedades no desarrolladas así como entre las mujeres inmigrantes de diferentes grupos étnicos obliga a reformular estas hipótesis vigentes. Las causas principales de los TCA tienen que ver con los sistemas de género que se han ido construyendo en la sociedad que se define con la hegemonía del capitalismo a partir de transformaciones de modelos de género que ya existían anteriormente, muy en particular, con la posición diferencial que las mujeres han ocupado respecto a los hombres en relación a la construcción de la identidad social y sexual, las imágenes corporales y, lo que es muy importante, en relación con las responsabilidades y valores asumidos respecto a la alimentación y las formas de ayuno.

Histórica y etnográficamente el ayuno femenino, en mayor proporción que el masculino, ha sido utilizado con diferentes finalidades: el no-comer, a menudo silencioso, para regular los recursos económicos del grupo, para socializar a sus miembros, para asegurar el control social. Cada una de estas finalidades tiene algún valor al describir una parte del todo. Cada una de ellas trabaja en un contexto elegido. La elección del contexto refleja los límites disciplinares o la ideología de los discursos dominantes. Lo que es definitivo para el autoayuno es el anteproyecto sociocultural cambiante en el que se produce. En nuestra cultura, las mujeres nutren, abastecen, educan, sirven... a los demás a través de la comida. Esta relación particular con la alimentación atraviesa el sistema de género, las relaciones interpersonales, la comunicación afectiva e, incluso, la aprehensión del riesgo, de tal forma que se convierte, si así se ha aprendido o se permite, en una herramienta con para expresar emociones, sentimientos y dificultades de índole diversa.

Por esta razón, el análisis de los trastornos alimentarios ha de hacerse desde la consideración de las diferentes formas que estas mismas mujeres practican el no comer o el hacerlo hasta no poder más. A muchas de estas mujeres les preocupa el peso o su cuerpo, como a la inmensa mayoría de mujeres, sin embargo no todas padecen trastornos del comportamiento alimentario. No para todas las mujeres el 
cuerpo es su cárcel o están obsesionadas por mantener las formas definidas socialmente. La hegemonía del ideal de delgadez corporal puede explicar, también en parte, el incremento de estos trastornos, pero no sólo o no siempre. Aunque los profesionales también son "seres comientes" -no sólo biológica sino socialmente-, no piensan en esa posibilidad. Apenas hablan, de sabores, paladar, placeres de la comida, o de sus sinsabores. Sólo aluden a la comida desde la salud y la enfermedad, y sólo hablan de la dieta como medio para obtener salud o adelgazar. Por si fuera poco, en el tratamiento de los trastornos alimentarios, se limitan a proponer a las pacientes una rutina dietético-nutricional difícil de cumplir en un contexto donde la pluralidad de actividades y de horarios fragilizan el seguimiento de las rutinas dietéticas, olvidan que las comidas son también formas de sociabilidad o placer y que el rechazo a los alimentos, el ayuno voluntario o el atracón pueden tener significados distintos.

\section{Conclusión}

La etnografía muestra que el trasfondo psicológico y biológico de los síntomas descritos por la biomedicina respecto a los trastornos alimentarios no se pueden desligar del contexto en el que han sido elaborados. Así pues, hay que considerar la compleja encrucijada sociocultural en la que se sitúan estas aflicciones con el objeto de disponer de elementos de juicio distintos que permitan redefinir algunos campos de intervención tanto en la esfera de la promoción de la salud y en general de la prevención, como en la intervención terapéutica y el seguimiento. Es primordial comprender cómo las mujeres perciben e interiorizan la comida, el cuerpo, la identidad de género y por qué dicha percepción puede dar respuestas más o menos lógicas y previsibles a las exigencias del entorno a través de las prácticas alimentarias extremas. En las dificultades expresadas las mujeres responden a parámetros generados desde fuera de ellas mismas, pero en relación con ellas mismas. El contexto cultural genera presiones de gran intensidad para todas, aunque sólo una parte acaban desarrollando conductas definidas clínicamente como psicopatológicas. Las factores individuales, tanto biológicos como psicológicos, pueden explicar parcialmente esta variabilidad, por más que en la mayoría de casos depende de cómo las personas perciben e interpretan los mensajes y los discursos de su entorno y de cómo los conciben. El conjunto de ideas y pensamientos se construye de diferentes maneras y en diferentes momentos según la situación y según los actores. De ahí el valor de incorporar aproximaciones analíticas que permitan dar cuenta de las relaciones entre subjetividades y contextos, y reflexionar sobre ellos.

\section{Agradecimientos}

Agradecemos muy sinceramente los distintos apoyos institucionales para desarrollar esta línea de investigación dentro del grupo consolidado de investigación "Grup 
d'Antropologia Social" de la URV. Han recibido financiación los siguientes proyectos: La incidencia de los factores socioculturales en los trastornos alimentarios de las mujeres: el caso de la anorexia nerviosa (2000-01). Instituto de la Mujer (61/00); Ambigüitat discursiva en la contrucció social de la feminitat. Evolució de les imatges culturals de les dones a través de la publicitat (2003). Institut Català de la Dona (U-4/03); Género, dieting y salud: una análisis transcultural de la incidencia de los trastornos del comportamiento alimentario entre las mujeres. Instituto de la Mujer I+D+I Exp. $\mathrm{n}^{\circ}$ 87/2003-2005; La cura del cos en cohorts de adolescents amb trastorn del comportament alimentari (2005). Fundación VECTEM (T050755)); Un análisis transcultural de los trastornos alimentarios en Catalunya: prácticas alimentarias, imágenes corporales y representaciones sociales de género entre los jóvenes. Observatori Català de la Joventut (AJOVE2005); Joves grassos, pobres joves!: formes de discriminació i ressistència a l'entorn de l'obesitat (2008 AJOVE 00017) y La emergencia de las sociedades obesogénicas o de la obesidad como problema social (I+D: CSO2009-07683, 2010-2012). 


\section{Bibliografía}

Appadurai, A. (2001) Après le colonialisme. Les conséquences culturelles de la globalisation, Paris: Payot.

Bados López, A. et al. (2002) "Eficacia y utilidad clínica de la terapia psicológica" Revista Internacional de Psicología Clínica y de la Salud, 2(3): 477-502.

Bell, R. (1985) Holy Anorexia, Chicago: The University of Chicago Press.

Bernal, M. (2011) Cuerpo, comida y migraciones: un análisis transcultural de los malestares alimentarios, Tarragona: Universitat Rovira i Virgili. Disponible en:http://www.tdx.cat/bitstream/handle/10803/34759/Tesis.pdf?sequence=1

Bordo, S. (1993) Unbearable Weight: Feminism, Western Culture, and the Body, Berkeley: University of California.

Brumberg, J. J. (1988) Fasting girls: the emergence of Anorexia Nervosa as a Moderns Disease, Cambridge: Harvard University Press.

Bulik, C. et al. (2007) "Anorexia nervosa treatment: A systematic review of randomized controlled trials" International Journal of Eating Disorders, 40(4): 310 320.

Chinchilla, A. (2003) Trastornos de la conducta alimentaria. Anorexia y bulimia nerviosas, obesidad y atracones, Barcelona: Masson.

Comelles, J. M. (1988) La razón y la sinrazón. Asistencia psiquiátrica y desarrollo del Estado en la España Contemporánea, Barcelona: PPU.

Counihan, C. (1999) The anthropology of food and body. Gender Meaning, and power, Nueva York: Routledge.

Di Nicola, V. (1990) "Anorexia Multiforme: selfstarvation in historical and cultural context” Transcultural Psychiatric Research Review, 27: 245-286.

Fernández-Aranda, F. y Turón, V. (1998) Trastornos de la alimentación: Guía básica de tratamiento en anorexia y bulimia, Barcelona: Masson.

Garner, D. y Garfinkel, P. (1980) "Sociocultural factors in the development of anorexia nervosa” Psychological Medicine, 10(4): 647-656.

Giordani, R. C. (2009) "O corpo sentido e os sentidos do corpo anoréxico" Rev. Nutr. 22(6): 809-821.

Gordon, R. (2000) Eating Disorders: Anatomy of a Social Epidemic, Oxford: Blackwell Publishers.

Gracia, M. (2002) "Los trastornos alimentarios como trastornos culturales” en M. Gracia (comp.), Somos los que comemos, Barcelona: Editorial Ariel.

Gracia, M. y Comelles, J. M. (eds.) (2007) No comerás. Narrativas sobre comida, cuerpo y género en el nuevo milenio, Barcelona: Editorial Icaria.

Gracia Arnaiz, M. (2009) "Learning to eat: The establishment of dietetic normality on eating disorders" Food, Culture and Society, 12(2): 191-216.

Gracia-Arnaiz, M. (2010) "Fat Bodies and Thin Bodies: Cultural, Biomedical and Market Discourses on Obesity” Appetite, 55(2): 219-225.

Gracia-Arnaiz, M. (2013) "Thou shalt not get fat: Medical representations and selfimages of obesity in a Mediterranean society” Health, 5(7): 1180-1189. 
Gracia-Arnaiz, M. (ed.) (2012) Pobres joves grassos! L’obesitat en les trajectòries juvenils, Tarragona: Servei de Publicacions URV.

Haro, J. (2000) “Cuidados profanos: una dimensión ambigua en la atención a la salud” en E. Perdiguero y J. M. Comelles (ed.), Medicina y cultura. Estudios entre la antropología y la medicina, Barcelona: Bellaterra.

Hepworth, J. (1999) The social construction of anorexia nervosa, Londres: Sage Publications.

Hine, C. (2004) Etnografía Virtual, Barcelona: Editorial UOC.

Katzman, M. y Lee, S. (1997) "Beyond body image: the integration of feminist and transcultural theories in the understanding of self starvation” Int J Eat Disord, 22(4):385-94.

Ledo, J. (2013) Usuarias de la red y TCAs: una análisis antropológico de los espacios, usos y narrativas virtuales, en M. Gracia (dir.), Universitat Rovira i Virgili, Tarragona.

Lester, R. (1995) "Embodied Voices: Women's Food Asceticism and the Negotiation of Identity" Ethos, 23(2): 187-222.

Lupton, D. (1994) Medicine as Culture, Londres: Sage Publications.

Martínez-Hernáez, A. (2008) Antropología Médica, Barcelona: Anthropos Editorial.

Menéndez, E. L. (1990) Antropología médica. Orientaciones, desigualdades y transacciones, México DF: CIESAS.

Moreno, J. L. (2010) Moral corporal, trastornos alimentarios y clase social, Madrid: CIS.

Prince, R. (1985) "The concept of culture-bound syndromes: anorexia nervosa and brain-fag" Social Science and Medicine, 21(2): 197-203.

Romaní, O. (1995) "Intervención comunitaria en drogodependencias. Etnografía y sentido común” Toxicodependencias, 2: 33-46.

Russell, G. F. M. (1985) "The changing nature of anorexia nervosa: An introduction to the conference”, Journal of Psychiatric Research, 19: 101-109.

Sirvent, C. (2009) "Fracaso, abandono y cumplimiento terapéutico" Norte de Salud Mental, 33: 51-58.

Toro, J. (1996) El cuerpo como delito, Barcelona: Editorial Ariel.

Toro, J. et al. (2005) "Assessment of sociocultural influences on the body shape model in adolescent males with anorexia nervosa” European Eating Disorders Review, 13(5): 351-359.

Turón, V. J. (1996) Trastornos de alimentación: anorexia nerviosa, bulimia y obesidad, Barcelona: Masson.

Van Dongen, E. (2000) "La forza dell'anoressia. Resistenza, energia e controllo" Revista della Società italiana di antropología médica, 9-10: 59-80.

Way, K. (1995) "Never Too Rich... Or Too Thin: The Role of Stigma in Social Constructions of Anorexia Nervosa”, en D. Maurer y Sobal, J. (eds.), Eating Agendas, Nueva York: Aldine de Gruyter.

Zafra, E. (2007) Aprender a comer: procesos de socialización y trastornos del comportamiento alimentario, Barcelona: Universidad de Barcelona. Disponible en:http://www.tdx.cat/bitstream/handle/10803 